Conclusion According to our findings; breech presentation, female sex, torticollis and multiple pregnancy were found to be the risk factors of this disorder. Infants with these risk factors should be investigated carefully for $\mathrm{DDH}$.

\section{P130 FIFTEEN-MINUTE CONSULTATION: THE INFANT WITH A SMALL HEAD}

${ }^{1}$ Joshua Alexander*, ${ }^{1}$ Nusrat Bholah, ${ }^{1}$ Naomi Okigbo, ${ }^{1}$ Ruth Devlin, ${ }^{1}$ Aisling Betts, ${ }^{1}$ Jack Healy, ${ }^{1}$ Sarah Higgins, ${ }^{1,2}$ Judith Meehan, 1,2,3,4Eleanor Molloy. 'Paediatrics, Trinity College Dublin, the University of Dublin, Dublin, Ireland; ${ }^{2}$ Tallaght University Hospital, Dublin, Ireland; ${ }^{3}$ Paediatrics, Coombe Women and Infants University Hospital, Dublin, Ireland; ${ }^{4}$ Neonatology, Our Lady's Children's Hospital, Dublin, Ireland

\subsection{6/archdischild-2019-epa.485}

Aims Microcephaly is a feature of multiple diseases, and has a vast array of aetiologies. Thus, identifying this condition clinically is only the start of establishing a diagnosis. Early assessment, analysis, and identification is vital to aiding the patient and family in managing the microcephaly itself, as well as future complications that may arise as a result. We aim to provide general practitioners, paediatricians and other health professionals, with a framework for initial approach to a patient presenting with a small head.

Methods An electronic review of literature on databases PubMed, Google Scholar, and Science Direct was performed searching Title/Abstract with the terms: [Microcephaly AND (assessment OR consultation OR examination)]. Previously published guidelines with information on initial approach to the microcephalic patient were also used in production of the proposed protocol.

Results We devised a protocol which helps clinicians to firstly diagnose microcephaly, and then separate primary microcephaly from secondary microcephaly. We then outlined potential investigations that should be undertaken to identify any underlying conditions. For primary microcephaly, specialised cytogenetic studies and gene sequencing and use of microarray can help to diagnose syndromes resulting in microcephaly. ${ }^{[i]}$ Secondary microcephaly is investigated through a comprehensive viral screen and range of biochemical blood tests to identify underlying causes. ${ }^{[i i]}$ The above methods are often combined with various imaging modalities, commonly MRI brain.

Conclusion As microcephaly is a clinical finding rather than a disease, a careful clinical history and examination are the cornerstones of the consultation, with findings guiding further investigations.

\section{REFERENCE}

1. Woods CG, Parker A. Investigating microcephaly. Archives of Disease in Childhood. 2013;98(9):707-713.

2. Michels T, Morris M, Myers T. Microcephaly. [internet]. 2009. Available from: https://core.ac.uk/download/pdf/62772193.pdf

\section{P131 'SEVERE NEUROLOGICAL IMPAIRMENT' - DOES THE TERM DEFINE AN AGREED COHORT FOR STUDY?}

1,2John Allen*, 1,2,3,4Eleanor Molloy, 1,2Denise McDonald. ${ }^{1}$ Trinity College Dublin, Dublin, Ireland; 'Tallaght University Hospital, Dublin, Ireland; ${ }^{3}$ Coombe Women and Infant's University Hospital, Dublin, Ireland; ${ }^{4}$ Our Lady's Children's Hospital Crumlin, Dublin, Ireland

10.1136/archdischild-2019-epa.486
Background Severe Neurological Impairment (SNI) is a term commonly used in the medical literature. There is no agreed definition of SNI. This limits opportunities for research into healthcare needs, treatment opportunities, resource planning and outcome. We reviewed the literature to establish consistency of use of the term and to place it in the context of other commonly employed terms used to describe children with severe, complex medical needs.

Methods A review of the literature was performed in October 2018. We searched the electronic database of PubMed and the Cochrane library. The search term 'Severe Neurological Impairment' was used. Animal studies were excluded but no other constraints were placed on the literature search. Fortytwo articles were included for full-text analysis. Definitions were analysed for common themes. Data were entered into an electronic spreadsheet and descriptive statistics were used determine frequency of the themes included in the various definitions.

Results Twenty-three of the papers reviewed included a definition of SNI, where the authors stated their interpretation of the use of the term SNI. In the other papers reviewed, the profile of the patients included by the authors provides insight into their understanding of the term. Motor impairment, intellectual disability, communication difficulties and increased care needs were included in the definition in $80 \%, 70 \%, 30 \%$ and $13 \%$ of papers respectively. Dependence on others for decision-making, chronicity and distinction between disorders of the central nervous system and peripheral nervous system were less frequently included.

Discussion This review demonstrates inconsistency in the use of the term Severe Neurological Impairment in the literature. Several other definitions exist to describe children with severe and complex medical conditions including Children with Special Healthcare Needs and Children with Medical Complexity. These definitions are often broad and include children with a wide variety of difficulties. Given their unique health and social care needs in the context of neurological impairment, an agreed definition distinguishing SNI from others would be helpful as a starting point to assess need and plan services.

Conclusions We have performed a comprehensive review of the literature and have shown that there is a wide variation in the use of the term Severe Neurological Impairment. A consensus-based definition of this term would be useful to facilitate future research.

\section{P132 EARLY FEEDING PROBLEMS AND ASSOCIATED FACTORS IN PREMATURE INFANTS}

${ }^{1}$ Ayşe Nur Coşkun*, ${ }^{2}$ Pınar Zengin Akkuş, ${ }^{2}$ Evin IIlter Bahadur, ${ }^{3}$ Hasan Tolga Çelik, ${ }^{2}$ Elif Nursel Özmert. ${ }^{1}$ Hacettepe University Faculty of Medicine, Department of Pediatrics, ANKARA, Turkey; ${ }^{2}$ Hacettepe University Faculty of Medicine, Department of Developmental an Behavioral Pediatrics, ANKARA, Turkey; ${ }^{3}$ Hacettepe University Faculty of Medicine, Department of Neonatology, ANKARA, Turkey

\subsection{6/archdischild-2019-epa.487}

Aim The aim of this study was to determine the frequency of feeding problems in premature infants and to investigate the relationship between growth, development and maternal depression.

Methods The study included 58 premature infants who were admitted to the Developmental Pediatrics Clinic between February-August 2018. Feeding problems obtained from the mothers by a questionnaire were classified as problems with oral- 